\title{
Energy Harvesting for Sensor Applications in the Energy Sector
}

\author{
Th. Keutel $^{1}$, T. Motl $^{1}$, S. Bdiri ${ }^{2}$, Ch. Viehweger ${ }^{1}$ and O. Kanoun ${ }^{1}$ \\ ${ }^{1}$ Chair for Measurement and Sensor Technology, Chemnitz University of Technology \\ thomas.keutel@etit.tu-chemnitz.de \\ ${ }^{2}$ National Engineering School of Sfax, Tunisia
}

\begin{abstract}
:
Because of the energy turnaround the power industry will be confronted with a massive restructuring. Only by an intelligent control prospectively it is possible to obtain a reliable energy supply. Wireless sensor systems can help to provide necessary information available as control variables. This paper presents a robust approach to use the electrostatic field of electrical equipment, for the supply of wireless sensor systems.
\end{abstract}

Key words: Energy harvesting, dc/dc converter, wireless sensing, power line monitoring

\section{Introduction}

A huge number of additional measured parameters of the operating grid [1], [2] became necessary to control the energy flow, the energy distribution or the main behavior of components of the transmission network in the future. Generally, instrumentation in such environments evokes heavy technical and monetary efforts. Within this context, a wireless sensor system accompanied by a self-organized power supply presents a promising alternative [3].

Analyzing system components of wireless sensor systems should be noted, wireless communication is already commercially available for wide spectra of applications [4]. Available methods for an autonomous power supply, like solar cells or thermo electrical converters, do not have a required reliability or availability [5]. Even though, in most cases the requirements for the rate of measurements foster the method using an ambient energy source [6]. More critical requirements for the system design of such a sensor approach results from the operation as an autonomous system. Most important, the system has to start-up reliably without any multiple charges. It's applicable as well to the generation of a control signal for necessary converter architecture [7], [8].

In this paper, a unique approach for an autonomous power supply will be proposed using the quasistatic field of electrical equipment of the grid.

\section{Energy Source Description}

The use of the quasistatic field of electrical equipment provides some advantages especially in the focused field of application.

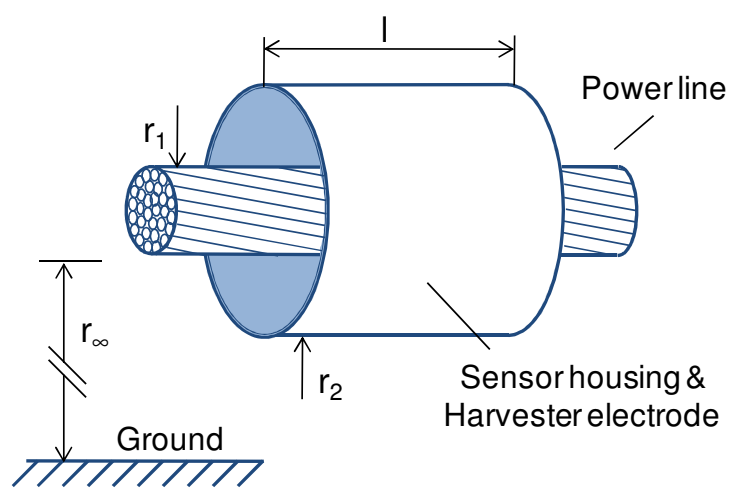

Fig. 1. Cylindrical structure to realize a capacitive voltage divider.

The quasistatic field is continuously available with a frequency of $50 \mathrm{~Hz}$ under high voltage. In case of the energy distribution, the voltage is constant regarding the voltage level of the distributing system. However, it is possible to use the electrostatic field by a capacitive structure which is shown in figure 1 as an exemplary for a cylindrical structure [9]. A separate electrode around the conductor realizes a capacitive voltage divider. It consists of a capacitance between the conductor and the electrode which is used later on as source and another capacitance between the electrode 
towards the ground. The voltage divides inversely proportional according to the ranges of the capacitors, as described in equation 1 ,

$$
C_{c y l .}=\frac{Q}{U_{E}}=\frac{\oint_{A} \vec{D} \cdot d \vec{A}}{\int \vec{E} \cdot d \vec{r}}=\frac{2 \pi \varepsilon_{0} l}{\ln \frac{r_{2}}{r_{1}}}
$$

The dependence on the small capacitance and the low frequency make the source impedance of the used capacitance leading to the range of some hundred mega ohms. An equivalent voltage source [10] is used to describe the capacitive voltage divider as shown in figure 2 . It also illustrates an analytical description of the electrical behavior that results into a normalized curve of the available power over the impedance. The technological realization is not currently possible when impedance coincide with a maximal available power. The challenge of using such high ohmic current sources is to develop converter architectures. That can realize adapted input impedances with high input voltage in order to enable the power demand of an energy autonomous system.

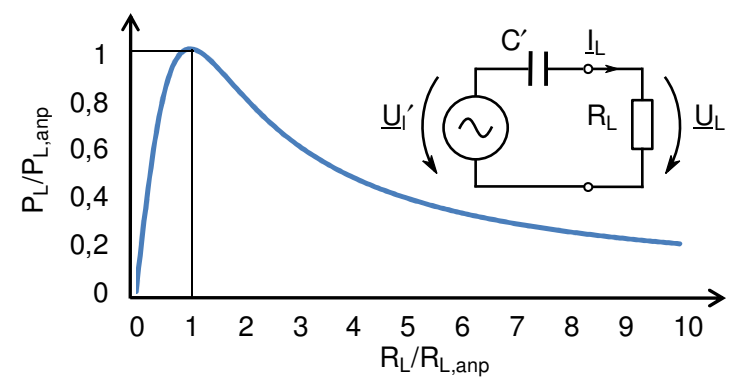

Fig. 2. Schematic of an equivalent voltage source normalized curve of the impedance depending available power.

\section{Electronic Design}

Various converter topologies were evaluated [11] in order to develop converter architectures as well as to realize sufficient input impedance. A forward converter topology was identified as a promising approach. This's because of the ability of making an adequate control signal at the first side. As described in equation 2, specific input impedance is realizable.

$$
Z_{\text {in }}=\frac{2 \cdot L}{T \cdot D^{2}} \cdot \frac{1}{1-\frac{U_{\text {out }}}{U_{\text {in }}}}
$$

D.. duty cycle

T.. period

In addition, as already mentioned, a reliable start-up mechanism a passive generation of a control signal are demanded for the operation as a power supply for an energy autonomous system, as show in figure 3 . Both components had to be placed at the primary side supplied by the input voltage of the system. A charge with less state at secondary side prevents the generation of a necessary control signal after long term interruptions.

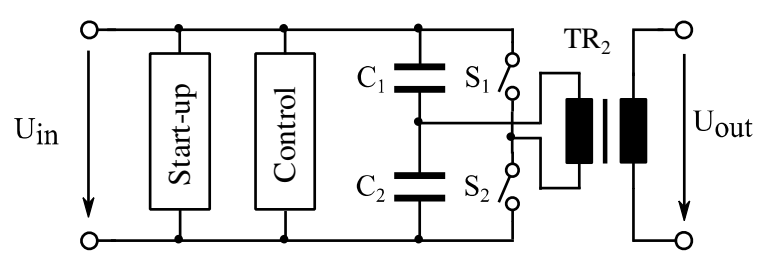

Fig. 3. Schematic of the forward converter topology including additional components.

\section{A. Control signal generation}

The figure 4 illustrates that minor requirements for the control signal characteristics of a forward converter enables the realization of the signal using a passive oscillating. The control transformer $\mathrm{TR}_{1}$ consists of one primary and two inverse secondary windings. Using saturation state of the core material, a periodical changing of the magnetization can be realized. Also it changes voltage signals at the secondary side of the transformer. These signals serve as control signal for the transistors $T_{1}$ and $T_{2}$. The switching frequency, as well as the voltage level of the $T R_{1}$ output signal is adjustable by winding and inductivity ratio.

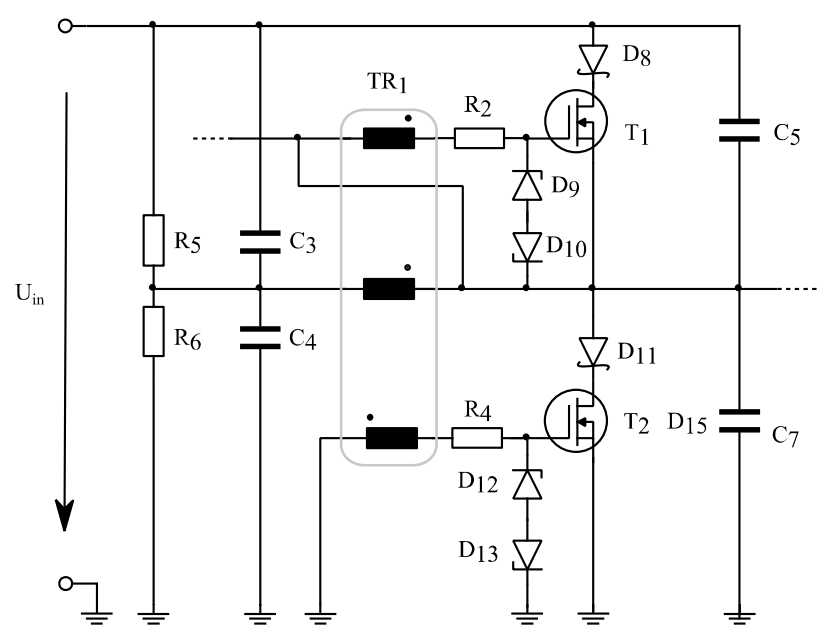

Fig. 4. Sectional schematic of the oscillating circuit including switching elements with separate gatevoltage protection

This process leads to a stable state by connecting the input voltage. A passive running start-up mechanism is necessary to start the oscillation and it represents the normal operation of the converter. 


\section{B. Start-up mechanism}

A reliable long term operation including source interruptions involves a passive start-up mechanism of the converter architecture. The operation at high voltage $(<500 \mathrm{~V})$ and low current $(\sim \mu \mathrm{A})$ represent the further design requirements. The influence on the input impedance and a passive behavior in normal operation is negligible.

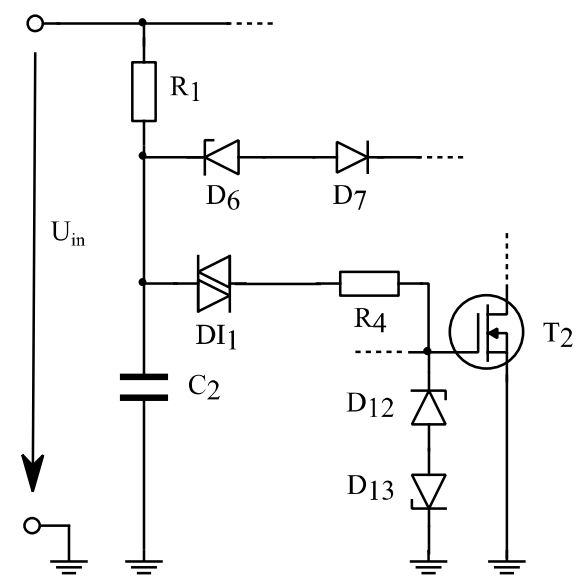

Fig. 5. Sectional schematic of the DIAC controlled start-up mechanism.

A major component of the start-up mechanism is the DIAC DI ${ }_{1}$ as shown sectional in figure 5. The capacitor $\mathrm{C}_{2}$ is slowly charged by a high ohmic resistor $R_{1}$ until the trigger voltage of $\mathrm{Dl}_{1}$ is reached and switched on. Afterwards the low side transistor $T_{2}$ will be activated and the oscillation will be running. There, where $D_{6}$ is limiting the voltage of $\mathrm{C}_{2}$ so that $\mathrm{Dl}_{1}$ doesn't reach the trigger voltage again in normal operation of the circuit.

\section{Impedance matching}

The transformer $\mathrm{TR}_{2}$ ensures the impedance matching as shown in figure 3. A specific capacitor separates the ac signal from a dc offset. In fact, a defined output signal is adjustable by the winding ratio of $\mathrm{TR}_{2}$. That transformer is responsible to realize a galvanic separation in order to protect sensitive components such as micro-controllers or RF-architectures.

\section{Circuit Evaluation}

A converter test prototype was evaluated in a laboratory test environment under high voltage power supply to benchmark the circuit. The main feature of the system is to function as a power supply for an energy autonomous system. The figure 6 illustrates the circuit characterization which is a sequence of the output signal of $\mathrm{TR}_{1}$. A switching frequency appears to be stable in the range of $\mathrm{f}_{\mathrm{s}}=200 \mathrm{kHz}$. An asymme- tric behavior occurs because of the usage of a third test winding at $\mathrm{TR}_{1}$ secondary side without voltage protection.

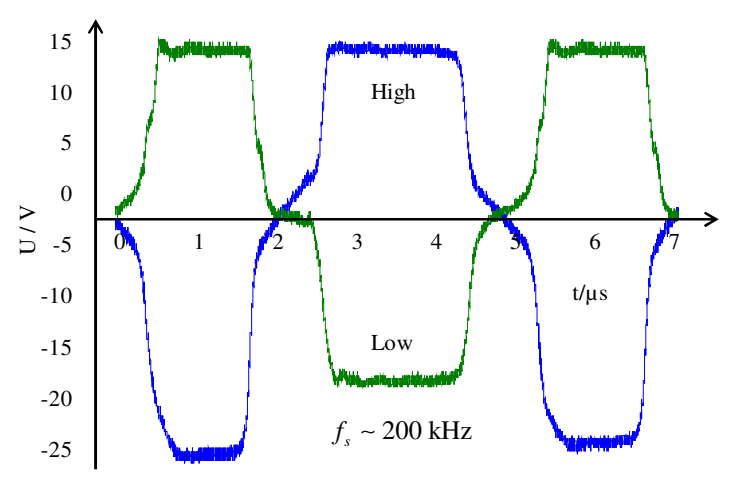

Fig. 6. Measured timing of the control transformer output signal.

The analysis in the high voltage test field was carried out using sensor housing as shown in figure 7 . The necessary electrode to build up the capacitive voltage divider is realized by a metalized inner side of the housing. Using a fiber-optic cable system, it is possible to make a characterization directly at a power line section under high voltage.

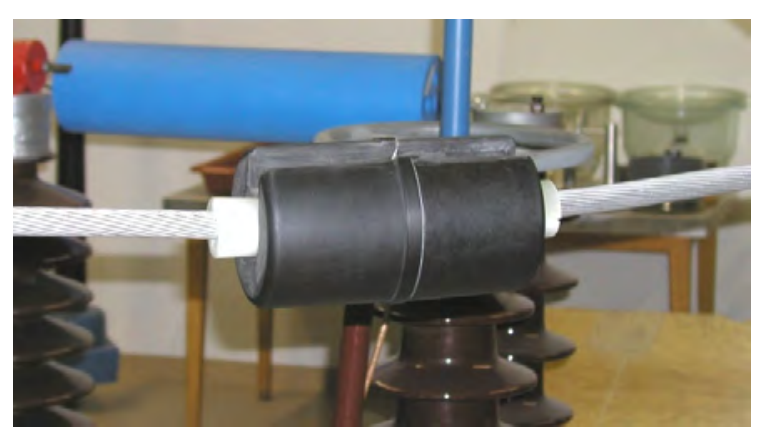

Fig. 7. Sensor housing for a laboratory test environment under high voltage

Figure 8 shows as an example, the charge timing of a storage capacitor $\mathrm{C}_{\mathrm{DLC}}$ at two different voltage levels $U_{0}$. This timing is used to evaluate the continuous output power $\mathrm{P}_{\mathrm{C}}$ of the converter using the equation 3 :

$P_{C}=\frac{1}{2} \cdot C_{D L C} \cdot \frac{\left(U_{D L C, N+1}^{2}-U_{D L C, N}^{2}\right)}{\Delta t}$

The measured curve indicates a discontinuous operation of the converter. This behavior appears if the charge of the source is too strong which leads to a voltage breakdown in the capacitor $\mathrm{C}_{1}$. It is charging again without converter operation.

By reaching the $\mathrm{Dl}_{1}$ trigger voltage the converter is starting up again. A higher input voltage 
leads to a higher frequency of this cycle and enables DC output power of approximately $\mathrm{P}_{\mathrm{c}} \sim 2 \mathrm{~mW}$. A continuous converter operation is realizable by increasing the input impedance of the converter as well as it increases the available output power. Because of a significant better behavior in the high voltage test field (for this method) in comparison to the behavior at a real overhead power line, the laboratory level of $60 \mathrm{kV}$ correlates approximately with the voltage level of a $110 \mathrm{kV}$ system.

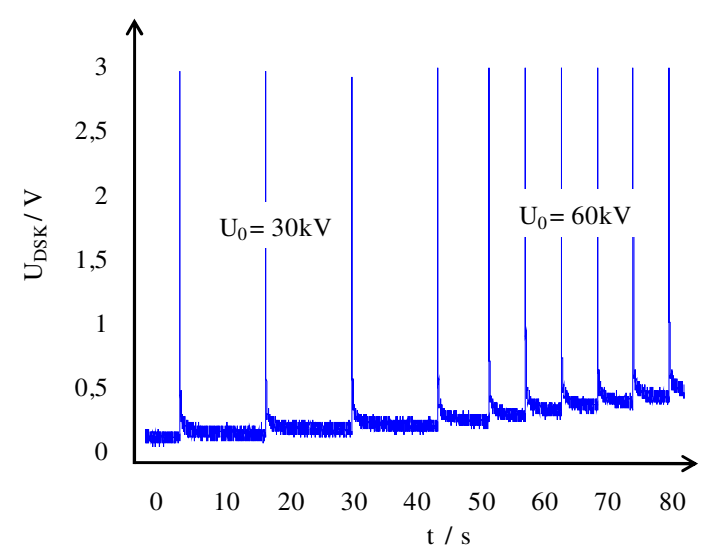

Fig. 8. Charge timing of a storage capacitor to evaluate the continuously output power.

\section{Conclusion}

Within this contribution a unique approach of an adapted forward converter topology is presented to use the electrostatic field of electrical equipment. The functionality was successfully demonstrated using a cylindrical sensor housing mounted on a power line section in a high voltage test field. A DC output power of approximately $P_{c}=2 \mathrm{~mW}$ was evaluated, already sufficient to supply specific wireless sensor networks. Optimization approaches to increase the available power were addressed. Finally there is a novel and robust method available to supply a wireless sensor system in the harsh environment of electrical equipment in the field of power generation and distribution. This method has the ability to reduce the efforts of instrumentation and especially for long term maintenance of such systems.

\section{References}

[1] VDE-Studie: „Dezentrale Energieversorgung 2020“, Energietechnische Gesellschaft im VDE (ETG), 2007

[2] Molderink, A., Bakker, V., Bosman, M.G.C., Hurink, J.L., Smit, G.J.M.: "Management and Control of Domestic Smart Grid Technology", IEEE Transactions on Smart Grid, vol.1, no.2, pp.109-119, doi: 10.1109/TSG.2010.2055904, Sept. 2010

[3] Kumar, P.R.:"Capacity, architecture, protocols, and sensing in wireless networks", IEEE Information Theory Workshop, pp. 469- 472, doi: 10.1109/ITW.2004.1405349, 24-29 Oct. 2004

[4] Akyildiz, I.F., Su, W, Sankarasubramaniam, Y. und Cayirci, E.: "Wireless sensor networks: a survey", in Computer Networks, Nr. 38, S. 393422, 2002

[5] Zhao, X., Keutel, T., Baldauf, M., Kanoun, O.: "Energy Harvesting for Overhead Power Line Monitoring", IEEE 9th International MultiConference on Systems, Signals and Devices, Chemnitz, Germany, ISBN: 9783981476644, March 20-23, 2012

[6] BMWi Studie: „Potenziale der Informations- und Kommunikations-Technologien zur Optimierung der Energieversorgung und des Energieverbrauchs (eEnergy)"; Studie für das Bundesministerium für Wirtschaft und Technologie, 2006

[7] Kanoun, O., Keutel, T.: "Energieautarke Sensorsysteme im Intelligenten Haus", ExpertVerlag, S. 150-160, ISBN 978-3-8169-2789-1, 2007

[8] Lazzarini Barnabei, A., Grassi, M., Pinna, D., Dallago, E., Malcovati, P., Ricotti, G.: "Integrated self-supplied system for environmental temperature sensing", IEEE Sensors, pp.12491252, doi: 10.1109/ICSENS.2011.6127308, 2831 Oct. 2011

[9] Zangl, H., Brasseur, G.:"A Feasibility Study on Autonomous Online Condition Monitoring of High-Voltage Overhead Power Lines", IEEE Transactions on Instrumentation and Measurement, Vol. 58, No. 5, May 2009

[10] Küpfmüller, K.:" Theoretische Elektrotechnik", 18. Auflage, Springer-Lehrbuch, ISSN 09377433, 2008G. Eason, B. Noble, and I. N. Sneddon, "On certain integrals of LipschitzHankel type involving products of Bessel functions," Phil. Trans. Roy. Soc. London, vol. A247, pp. 529-551, April 1955

[11] Keutel,T.: "Energiewandlung aus hochohmigen kapazitiven Stromquellen für die Versorgung drahtloser Sensorik aus dem elektrostatischen Feld von Hochspannungsleitungen" Dissertationsschrift, Chemnitz, 2012 noch nicht Veröffentlicht 